\title{
INTEGRATIONSHERAUSFORDERUNGEN UND DIE Bedeutung Sozialer Netzwerke
}

\author{
Marie LEHNER, Institut für Stadt- und Regionalforschung, Österreichische \\ Akademie der Wissenschaften, Wien \\ Mona Röнm, Department Politikwissenschaft und Soziologie, Universität \\ Salzburg
}

\section{INHALT}

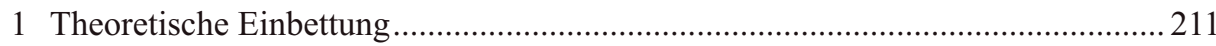

Der politische und rechtliche Integrationsbegriff im österreichischen Kontext...213

2 Das Zusammenwirken sozialer Kontakte und struktureller Integration am Beispiel afghanischer Geflüchteter in Österreich .............................................2.215

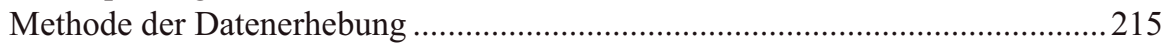

Afghan/inn/en in Österreich - ein Überblick über die aktuelle Situation .............216

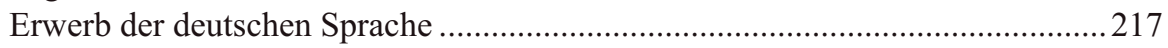

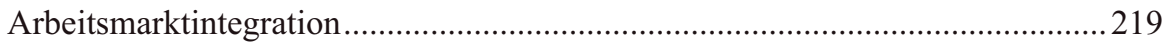

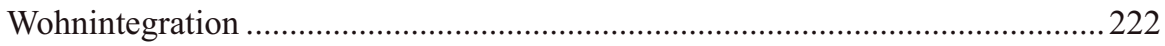

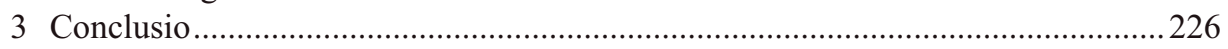

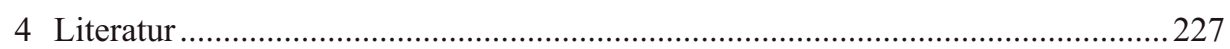

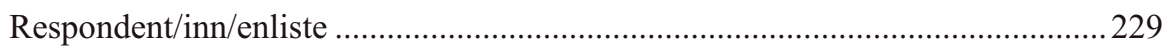

Sich ein Leben in einer unbekannten und neuen Gesellschaft aufzubauen stellt Jede und Jeden vor große Herausforderungen. Die Voraussetzungen, die Individuen für einen solchen Start mit sich bringen, variieren stark und sind abhängig von den ökonomischen, politischen, sozialen und kulturellen Gegebenheiten im Herkunftsgebiet. Um als gleichwertiges Mitglied in einer neuen Gesellschaft akzeptiert zu werden, haben migrierte Personen Anpassungsprozesse zu unterlaufen, (die u.a. vom Grad des kulturellen Unterschieds zur Aufnahmegesellschaft abhängig sind). Das Aufnahmeland gibt dabei die politischen und rechtlichen Rahmenbedingungen vor, die neben der individuellen Bereitschaft zur Eingliederung von Seiten der Aufnahmegesellschaft, sowie von Seiten der migrierten Personen, als ausschlaggebender Faktor für den Grad der Integ- 
ration bestimmt werden können. Dabei erschweren eine Entkontextualisierung aus der gewohnten sozialen und kulturellen Umgebung, traumatische Erfahrungen während der Flucht, sowie Vorurteile und Diskriminierungserfahrungen innerhalb der Mehrheitsgesellschaft, ein Zurechtfinden in der neuen Lebenssituation. Stresssymptome begleiten somit oftmals Akkulturationsprozesse und nehmen Einfluss auf das Erlernen sozialer Verhaltensweisen, die für eine Einbindung in die Mehrheitsgesellschaft des neuen kulturellen Umfelds erforderlich sind (BARRY 1997). Dabei werden jene Individuen/ Gruppen als besonders herausfordernd betrachtet, die einen großen kulturellen Unterschied zur Aufnahmegesellschaft aufzeigen. Ein sozialer Austausch zwischen Personen aus der Aufnahmegesellschaft und der sich integrierenden Individuen ist somit notwendig, um sich in einer neuen Gesellschaft einbinden zu können. Die soziale Interaktion kann dabei nicht nur auf Personen aus der bestehenden Mehrheitsgesellschaft reduziert werden. Entgegen einem aktuellen öffentlichen Diskurs, der bestehende ethnische Gemeinschaften als Hindernis bei Adaptionsprozessen thematisiert, bieten intraethnische Netzwerke Schutzräume und Hilfestellung für ankommende Individuen, besonders dann, wenn darin ein hohes Maß an ökonomischen und sozialen Ressourcen vorhanden ist (FARWICK 2009). Es stellt sich somit die Frage, wie migrierte Gruppen/ Individuen (hier: geflüchtete Afghan/inn/en) in das gesellschaftliche Zusammenleben des Aufnahmelandes (hier: Österreich) eingebunden und welche integrativen Herausforderungen damit in Beziehung gesetzt werden. In Bezug auf die rezente Fluchtbewegung nach Europa - Österreich im Jahr 2015 (siehe Abschnitt: Afghan/inn/en in Österreich - ein Überblick zur aktuellen Situation) wird in diesem Beitrag, im Rahmen des Forschungsprojektes LODA „Loslassen - Durchstehen - Ankommen“" , der Zusammenhang zwischen staatlichen Erwartungen an geflüchtete Afghan/inn/en - anhand gesetzlich festgelegter Integrationsmaßnahmen - und den realen Umsetzungsmöglichkeiten dargestellt. Unser Ziel ist es, am Beispiel afghanischer Geflüchteter in Österreich, die Relevanz der sozialen Kontakte für die Umsetzung erster Schritte einer strukturellen Integration zu veranschaulichen.

Um einen Überblick zu schaffen, werden im ersten Teil dieses Beitrags die verwendeten Begrifflichkeiten im Integrationskontext thematisiert, sowie anschließend auf die gesetzlich verankerten Integrationsmaßnahmen in Österreich Bezug genommen.

Der zweite Teil widmet sich der Analyse des empirischen Materials. Dabei gehen wir zunächst auf die Situation afghanischer Geflüchteter in Österreich allgemein ein und beschäftigen uns im Folgenden genauer mit der Frage nach der Tragweite sozialer

\footnotetext{
Das Projekt LODA „Loslassen - Durchstehen - Ankommen“ ist ein interdisziplinäres Forschungsprojekt, das im Rahmen des ÖAW-Netzwerks ROR-N im Zeitraum März 2017 bis Februar 2019 durchgeführt wird. Ziel des Projekts ist es, mit wissenschaftlichen Daten einen Beitrag zur Versachlichung des öffentlichen Diskurses über Geflüchtete zu leisten sowie Politikempfehlungen zu entwickeln. Insgesamt wurden 100 narrative Interviews mit Syrer/inne/n, Iraker/ inne/n und Afghan/inn/en sowie 14 Expert/inn/eninterviews geführt. Die beiden Autorinnen waren bzw. sind als Projektmitarbeiterinnen am Institut für Stadt- und Regionalforschung tätig. Mehr Infos: <www.oeaw.ac.at/isr/forschung/ag-urbane-transformation/loslassen-durchstehenankommen/>
} 
Kontakte bei strukturellen Integrationsprozessen. Der Fokus in Bezug auf strukturelle Eingliederung liegt dabei auf drei Bereichen, die sich als besonders relevant herausgestellt haben: der Erwerb der deutschen Sprache als förderliche Basis für eine strukturelle Integration, die Arbeitsmarkt- sowie die Wohnintegration. Abschließend werden die Ergebnisse zusammengefasst.

\section{Theoretische Einbettung}

Die Frage der Integration wird in den öffentlichen und medialen Diskursen oftmals im Zusammenhang mit Migrationsbewegungen thematisiert. Während sich Migration mit der Wanderung von Menschen beschäftigt, sowohl innerhalb der Staatsgrenzen als auch grenzüberschreitend, stellt sich die Frage der Integration dann, wenn die Seite der Einwanderung und der Aufnahmekontext betrachtet werden sollen (ELwERT 2015). Der Integrationsbegriff, der hier im Allgemeinen die Eingliederung von Migrant/inn/ en in verschiedene Bereiche der Aufnahmegesellschaft bezeichnet (wie etwa Sprache, Kultur, Arbeitsmarkt, soziale Beziehungen u.v.m.) und erweitert auf die Herstellung eines zusammenhängenden Ganzen einzelner Elemente Bezug nimmt (vgl. ReINHOLD 2000, p. 299), wurde von zahlreichen Vertreter/inne/n der Migrationsforschung immer wieder kritisiert bzw. neu definiert. Dabei werden Begrifflichkeiten, wie Akkulturation, Assimilation und Adaption oftmals synchron verwendet und einem Integrationsbegriff gleichgesetzt, obwohl sie unterschiedliches bedeuten. Während Adaption die individuelle Strategie der Migrant/inn/en bei Eingliederungsprozessen beschreibt (HAN 2016), definiert Akkulturation eine Anpassung migrierter Individuen an kulturelle Gewohnheiten der Einwanderungsgesellschaft (z.B. im Bereich der Sprache und der sozialen Verhaltensweisen). Diese Form der impliziten Angleichung bezieht sich in vielen Fällen jedoch nicht nur auf kulturelle Gewohnheiten, sondern es kommt darüber hinaus zu einer umfassenderen Assimilation - einer Reduktion bzw. Verschwinden von Grenzen und Unterschieden zwischen sozialen/ethnischen Gruppen ${ }^{2}$ (HaNs 2016). Wenngleich Assimilation und Integration nicht gleichbedeutend sind, können bestimmte Bereiche der Integration eine Assimilation voraussetzen (z.B. Sprache). ${ }^{3}$ Es ist also wichtig im Rahmen migrations- und integrationsspezifischer Analysen, und somit auch in diesem Beitrag, Begrifflichkeiten und deren Zusammenhänge genauer zu erläutern. In diesem Zusammenhang kann auf Esser (2001) Bezug genommen werden, der Integration als gesamtgesellschaftliche Konzeption versteht und basierend auf LocKwoods (1964) zweistufiger Konzeption, Systemintegration und Sozialintegration gegenüberstellt. Während unter Systemintegration die Integration des Systems (Prozess ohne Rücksichtahme einzelner Akteur/inn/e/n) einer Gesellschaft als Ganzheit zu verstehen

\footnotetext{
Das Gegenteil zu Assimilation wäre Segregation. Hier kommt es nicht zu einer Angleichung, sondern die Unterschiede sozialer Gruppen bleiben dauerhaft bestehen (Hans 2016).

3 Da jene Begrifflichkeiten (Akkulturation, Assimilation, Adaption) unterschiedlichsten Interpretationen und Definitionen obliegen, wird hier auf die allgemeine Definition von Hans (2016) Bezug genommen.
} 
ist (Makroebene), bezieht sich die Sozialintegration auf die Integration der einzelnen Akteur/inn/e/n in das gesellschaftliche Zusammenleben (Mikroebene) (Esser 2001). Gesellschaften können daher am ehesten als integriert gelten, wenn sich System- und Sozialintegration gegenseitig ergänzen und verstärken (vgl. Anhut/HeitMeYer 2000). Verwendet man den Integrationsbegriff nun bei Migrant/inn/en, so umfasst dieser das Miteinbeziehen der Akteur/inn/e/n in die Aufnahmegesellschaft unter den Voraussetzungen der Gewährung von Rechten, des Erwerbs von Sprachkenntnissen, der Beteiligung am Arbeitsmarkt und im Bildungssystem, der sozialen Akzeptanz der Akteur/ inn/e/n, einer Beteiligung am öffentlichen und politischen Leben, sowie einer emotionalen Identifikation der Ankommenden mit dem Aufnahmeland. Dabei unterliegen die genannten Formen und Dimensionen der (Sozial-)Integration kausalen Beziehungen und bestimmen den Grad der Integration (Esser 2001). So können drei Sphären der Integration unterschieden werden, die strukturelle, die soziokulturelle und die personale Integration (siehe auch WETS 2006). Strukturelle Indikatoren werden dabei oftmals herangezogen, um den Grad der Integration zu messen. Die Doppeldeutigkeit des Integrationsbegriffs wird hier erkenntlich - neben einem soziologisch determinierten Fachterminus beinhaltet jener auch politische Zielvorstellungen. Die Politik dekretiert deshalb Integrationsmaßnahmen, die dazu beitragen sollen, Migrant/inn/en in strukturelle Funktionsbereiche einzugliedern. Die Frage der Integration bezieht sich somit auch immer auf Adaptionsprozesse der migrierten Individuen/Gruppen an die neue Umgebung, die von Seiten der Politik über geforderte rechtliche und ökonomische Standards gemessen werden - strukturelle Indikatoren werden oftmals herangezogen, um den Grad der Integration zu messen. Ager und StRAng (2008) kritisieren ein Messen von Integrationsleistungen über Indikatoren (sogenannte „,makers“ wie z.B. Erwerbsarbeit, Wohnung oder Weiterbildung), da diese zugleich Ausdruck von, sowie Instrument zur Integration sein können und somit den Charakter von Integrationsanforderungen erreichen können. Der Zugang zu solchen politisch gesetzten Maßnahmen ist jedoch mit bestimmten Rechten (z.B. Asylstatus) verbunden, die die Grundlage für eine individuelle Integrationsleistung bilden. Der österreichische Staat zum Beispiel lehnt eine Integration während der Dauer des Asylverfahrens ab. Die Rechte, die einen Zugang zu Integrationsmaßnahmen und somit auch das Miteinbeziehen auf institutioneller Ebene gewährleisten, entfallen demnach für Asylwerber/innen (ROSENBERGER 2011) - dies wird im folgenden Absatz noch näher erläutert. Obwohl der Grad der Inklusion in strukturelle Funktionsbereiche vom Status der jeweiligen Person abhängig ist, ist der Zugang zu bestimmten Rechten und gegebenen Integrationsmaßnahmen keine Gewähr für eine strukturelle Einbindung (vgl. AsChAUER/GANN 2017). Sozioökonomische und politische Benachteiligungen ethnischer Minderheiten (hier: Afghan/inn/en) erschweren u.a. eine Teilnahme am gesellschaftlichen Leben des neuen Umfelds. Oftmals stellen z.B. gegebene Wohnverhältnisse, fehlende Anerkennung der Qualifikationen am Arbeitsmarkt, Vorurteile und Diskriminierung von Seiten der Aufnahmegesellschaft, eine Barriere für die Einbindung in strukturelle Integrationsprozesse dar und offenbaren eine begrenzte Wirkung politisch gesetzter Maßnahmen. 
Ausschlaggebend für eine Analyse des Zusammenspiels von sozialer und struktureller Integration ist insbesondere der Blickwinkel, Migrant/inn/en nicht als passive Unterstützungsempfänger/innen zu betrachten, sondern ihre aktive Rolle in sozialen Netzwerken wahrzunehmen (Williams 2006). Dabei sind die sozialen Beziehungen je nach Kontext, Intensität, Nutzen und Dauerhaftigkeit variabel (siehe WiLliams 2006; Granovetter 1973) und ermöglichen es Individuen oder Kollektiven, bestimmte Ressourcen aus dem sozialen Netzwerk ab dem Zeitpunkt der Ankunft und ersten Kontaktaufnahme zu nutzen (DeindL 2005; KohlBacher 2017). Die Beziehung zu unterschiedlichen Personengruppen ermöglicht es Individuen/Gruppen während des „Prozesses des Fußfassens“ von unterschiedlichen Ressourcen (z.B. Unterstützung, Informationen etc.) Gebrauch zu machen. Dabei entsteht soziales Kapital (DeINDL 2005; LiN 2001; HaUg 2007), das sowohl die soziale Einbettung jener Individuen, als auch deren Fortschritt im Integrationsprozess am Zielort bestimmt. Migrant/inn/en sollten deshalb nicht als isolierte Akteur/inn/e/n betrachtet werden, sondern deren Eingebundenheit in soziale Netzwerke muss besondere Berücksichtigung erfahren, um sowohl zwischenmenschliche Verbindungen zu erkennen, als auch den daraus entstehenden Nutzen dieser Ressource zu analysieren (Boyd/NowAK 2012). Daher ist es notwendig die sozialen Beziehungen, die als ein Element den Sozialstaat konstituieren, zu den gegebenen politischen und gesetzlichen Rahmenbedingungen in Bezug zu setzen. Der viel diskutierte Integrationsbegriff zeigt sich somit abhängig von einem zeitlichen und örtlichen Bezugsrahmen, involviert unterschiedliche Interessen und Akteur/inn/e/n und verlangt eine gesamtgesellschaftliche Konzeption, da er sowohl Zugewanderte, als auch die bestehende Aufnahmegesellschaft gleichermaßen inkludiert (AGER/Strang 2008; Ager/Eyber 2002; CAstles et al. 2002; Musterd 2003).

\section{Der politische und rechtliche Integrationsbegriff im österreichischen Kontext}

Der österreichische Nationalstaat, der in Asylagenden an die Europäische Union gebunden ist, leitete im Jahr 2010 eine neue Integrationsstrategie ein und verabschiedete im Ministerrat den „Nationalen Aktionsplan für Integration“. Grundlage des sogenannten NAP.I bilden, bei dauerhaftem Aufenthalt in Österreich, Integrationsbestrebungen vom ersten Tag an. Aufgrund der rasant angestiegenen Zahlen an Schutzsuchenden im Jahr 2015 wuchs der Bedarf an einer institutionsübergreifenden Strategie, die vom Bundesministerium für Europa, Integration und Äußeres unter der Mitarbeit des Expertenrats für Integration mit der Erstellung eines 50-Punkte-Plans beantwortet wurde (EXPERTENRAT FÜR INTEGRATION 2016). Dieses Grundlagendokument gilt seit Jänner 2016 als bundesweite Integrationsstrategie für Asyl -und Schutzberechtigte in Österreich und baut auf den sieben Handlungsfeldern des NAP.I auf, die darin in 50 Unterpunkten erläutert werden (enthalten sind darin Themenbereiche wie Sprache und Bildung, Arbeit und Beruf, Rechtsstaat und Werte, Interkultureller Dialog, Gesundheit 
und Soziales, Sport und Freizeit sowie Wohnen und die regionale Dimension der Integration). Die angeführten Punkte sollen als Anleitung dienen, dem Ziel der Integration von Asyl- und Schutzberechtigten, nämlich einer raschen Selbsterhaltungsfähigkeit sowie einem aktiven Bemühen um das eigene Fortkommen und Einbringen in die österreichische Gesellschaft, näher zu kommen. Zur Umsetzung der aus allen Lebensbereichen entnommenen Maßnahmen ist eine Zusammenarbeit von Bund, Ländern, Gemeinden, Sozialpartner/innen und der Zivilbevölkerung gefragt (BMEIA 2015; EXPERTENRAT FÜR INTEGRATION 2017). Dabei versteht die österreichische Regierung Integration als linearen Prozess und priorisiert eine rasche strukturelle Einbettung mittels gesetzlich verankerter Maßnahmen. Diese Maßnahmen beinhalten Schritte zur Arbeitsmarktintegration (AuBG - 17.07.2016; IJG - 01.09.2017) sowie Spracherwerb bzw. Alphabetisierung in lateinischer Schrift und die Vermittlung von Werte- und Orientierungswissen (IntG - 09.06.2017). Für die Umsetzung und Ausführung der Integrationsmaßnahmen sind staatliche Institutionen wie der ÖIF (Österreichische Integrationsfond) und das AMS (Arbeitsmarktservice) verantwortlich, die sich im Verantwortungsbereich des Bundesministeriums für Europa, Integration und Äußeres (BMEIA) als auch des Bundesministeriums für Arbeit, Soziales, Gesundheit und Konsumentenschutz (BASGK) befinden (ÖIF 2017). Die österreichische Regierung beschränkt jedoch den Zugang zu den gesetzlich festgeschriebenen Maßnahmen auf asyl- und subsidiär schutzberechtigte Personen. So sind nach Erhalt des Schutzstatus jene Menschen rechtlich im Allgemeinen den Österreicher/inne/n gleichgestellt und verpflichtet „Integrationsleistungen“ $\mathrm{zu}$ erfüllen, sowie aktiv am Integrationsprozess mitzuwirken (Integrationspflicht, §1 Abs.1, IntG). Personen ohne Status können von den geforderten Integrationsmaßnahmen, wie Sprachkursen, Arbeitsmarktintegration und der Vermittlung von Werte- und Orientierungswissen keinen Gebrauch machen und sind hinsichtlich ihrer Rechte stark eingeschränkt. So ist es Asylwerbenden nach dreimonatiger Asylverfahrensdauer gestattet, folgende Tätigkeiten legal auszuüben (während die unselbständige Erwerbstätigkeit für Asylwerbende stark eingeschränkt ist, wird eine selbständige gestattet): Personen im Verfahren dürfen einer vorübergehenden, d.h. nicht auf Dauer ausgerichteten, Tätigkeit nachgehen - diese Hilfstätigkeit soll einen gemeinnützigen Charakter aufweisen. Des Weiteren ist es Asylwerbenden gestattet, im Bereich der Saisonarbeit einer befristeten Beschäftigung nachzugehen, mittels Dienstleistungschecks in Privathaushalten (Haus-, Garten- und Kinderbetreuung) tätig zu sein bzw. einer Lehrtätigkeit in Mangelberufen nachzugehen - dies soll dem Abruf aus der Schwarzarbeit dienen. Auch dürfen Volontariate, Ferialund Berufspraktika ausgeübt werden, sofern das AMS eine Anzeigebestätigung erteilt. Laut rezenten politischen Entwicklungen in Österreich will die Bundesregierung nun die Lehre bei Mangelberufen für junge Asylwerbende abschaffen (AsYLKoordination Österreich 2017; LANDESKorRespondenz Medieninfo 2017; AMS 2017; Szigetvari 2018; DERSTANDARD.AT).

Die angeführten rechtlichen Rahmenbedingungen, die aufgrund schwankender Zahlen an Geflüchteten und politischen Umstrukturierungen einem ständigen Wandel unterworfen sind, zeigen auf, dass bereits im Vergleich zwischen Personen mit Status 
und Asylwerber/inne/n die Möglichkeiten einer strukturellen Einbindung erschwert sind und die Teilhabe an der österreichischen Gesellschaft nur sehr beschränkt möglich ist. Jedoch sind selbst innerhalb der Gruppe der Asyl- und subsidiär Schutzberechtigten bei Afghan/inn/en vermehrte Schwierigkeiten im Rahmen der strukturellen Einbeziehung ersichtlich. Wie unsere empirischen Daten belegen, sind im Besonderen in den Bereichen Wohnen, Arbeit und Sprache, auch bei vorhandenem Zugang zu staatlichen Maßnahmen, Probleme bezüglich der strukturellen Einbindung ersichtlich. Folglich kommt dem Zugang und der Qualität sozialer Kontakte eine Brückenfunktion zu, um am gesellschaftlichen Leben in Österreich Anteil nehmen und „Fuß fassen“ zu können.

\section{Das Zusammenwirken sozialer Kontakte und struktu- reller Integration am Beispiel afghanischer Geflüch- teter in Österreich}

Nach dem Überblick über die Begrifflichkeiten von (struktureller und sozialer) Integration, deren Bedeutung im politischen Kontext in Österreich sowie über die damit zusammenhängenden verpflichtenden Integrationsmaßnahmen zur Förderung struktureller Integration wird im Folgenden anhand des empirischen Materials des Forschungsprojektes LODA die Relevanz sozialer Kontakte im Rahmen der strukturellen Integrationsprozesse veranschaulicht.

\section{Methode der Datenerhebung}

Um die Situation geflüchteter Afghan/inn/en hinsichtlich erster Schritte des Ankommens und Fußfassens in Österreich erklären zu können, wurden zwischen Oktober 2017 und Dezember 2018, 33 leitfadenorientierte Interviews mit afghanischen Respondent/inn/en sowie weitere 14 mit Expert/inn/en im Migrations- und Integrationsbereich durchgeführt. Um die Vielfalt der unterschiedlichen Lebensrealitäten darstellen zu können, wurde beim Sampling der Interviewpartner/innen insbesondere auf die Heterogenität hinsichtlich Alter, Bildungsstatus, ethnischer Zugehörigkeit, Geschlecht, Aufenthaltsstatus sowie Dauer des Aufenthalts in Österreich geachtet.

Die Kontaktaufnahme zu den Interviewpartner/inne/n erfolgte auf unterschiedliche Weise. Zunächst wurden bestehende Kontakte aus vorangegangenen Studien sowie insbesondere die sozialen Netzwerke der afghanischen Interviewer/innen zur Akquirierung von Gesprächpartner/inne/n herangezogen. Diverse Gatekeeper/innen und Multiplikator/innen der afghanischen Community unterstützten bei der Anwerbung neuer Respondent/inn/en. Auf diesem Weg wurde dem Interviewer/innenteam ein gewisser Vertrauensvorschuss entgegengebracht, der vor allem bei sensiblen Gesprächsthemen notwendig ist (vgl. KRUSE 2015). Kombinierte Verfahren zum Kontaktaufbau 
erwiesen sich dahingehend als besonders vorteilhaft. Im weiteren Verlauf der Datenerhebung wurden, basierend auf den bereits aufgebauten Kontakten, anhand des Schneeballsystems weitere Gesprächspartner/innen akquiriert (vgl. LEWIS-BECK/BYRMAN/LiAO 2004). Zusätzlich und um der des Schneeballsamplings zugesprochenen Gefahr nur eine bestimmte Personengruppe zu erreichen, entgegenzuwirken, begaben sich die Projektmitarbeiter/innen ins Feld, nahmen an Veranstaltungen teil und verbrachten Zeit mit potentiellen Gesprächspartner/inne/n. Ein weiterer Vorteil liegt hier in der Möglichkeit persönliche informelle Gespräche zu führen und damit eine Vertrauensbasis aufzubauen. Alle Interviews wurden je nach Präferenz der Interviewpartner/innen auf Farsi, Dari oder Deutsch durchgeführt.

Die Expert/inn/en inkludieren Personen aus unterschiedlichen Bereichen, die direkt oder indirekt mit Geflüchteten zusammenarbeiten, wie z.B. Vertreter/innen aus der Politik und Administration, Sozialarbeiter/innen, Vertrete/innen von NGOs sowie Personen, die im Gesundheitswesen oder Bildungsbereich tätig sind.

Der Interviewführung folgend, wurden Gesprächsprotokolle erstellt, die über den Interviewverlauf Aufschluss gaben und zusätzlich nicht-digital aufgezeichnete Informationen beinhalteten. Gemeinsam mit den Transkriptionen der Interviews bildeten sie die Datenbasis für die anschießende Auswertung, die mithilfe des Computerbasierenden Analysetools Atlas.ti durchgeführt wurde. Dabei wurde zunächst eine Kategorienliste (Index-Codes) erstellt und anhand dessen das Datenmaterial gesichtet. Im weiteren Analyseverlauf wurden der Kategorienliste In-Vivo Codes (Kategorien, die sich direkt aus dem Material ergeben) hinzugefügt (vgl. Dewalt/Dewalt 2011). Eine Interpretation der Interviews (Qualitative Inhaltsanalyse) anhand der relevanten Themen als auch ein In-Beziehung-Setzen der Textstellen und Kontextinformationen über die Interviewpartner/innen, wurde unter Einbindung theoretischer Konzepte als letzter Schritt der Datenanalyse durchgeführt.

\section{Afghan/inn/en in Österreich - ein Überblick über die aktuelle Situation}

Österreich ist neben Deutschland und Schweden eines der wichtigsten europäischen Zielländer für Geflüchtete aus Afghanistan. Im Jahr 2015 repräsentierten sie mit 25.500 Personen, neben Geflüchteten aus Syrien und dem Irak, die größte Gruppe, die in Österreich Asyl beantragte. Derzeit leben ca. 46.000 Menschen mit afghanischer Staatsbürgerschaft in Österreich, mehrheitlich in der Bundeshauptstadt Wien, gefolgt von den Bundesländern Oberösterreich und der Steiermark (Statistik Austria 2018). Eine Ankunft in Österreich konfrontiert viele Afghan/inn/en mit großen Herausforderungen. Herkunftsort (Afghanistan, Pakistan, Iran), Alter, Geschlecht, Bildungsstand etc. bezeichnen nur einige Variablen der sehr heterogenen Gruppe, die Einfluss auf individuelle Integrationsprozesse nehmen. Jene sind neben den reglementierten Integrationsmaßnahmen auch von einer Aufnahme- bzw. Eingliederungsbereitschaft der öster- 
reichischen Bevölkerung und Regierung abhängig. Öffentliche und politische Diskurse trugen dazu bei, dass Afghan/inn/en das Image einer Personengruppe auferlegt wurde, die ,[...] in einer zutiefst patriarchalischen Gesellschaft mit archaischen Wertvorstellungen aufgewachsen ist und in einem westeuropäischen Land kaum integrierbar ist [...]“ (FREY 2016, derstandard.at). Insbesondere junge, männliche Afghanen sind mit Vorurteilen konfrontiert und werden innerhalb medialer Stereotypisierung als aus ,[...] frauenverachtenden Gesellschaften mit einem kranken Verhältnis zur Sexualität [...]““ (RAUSCHER 2016, derstandard.at) stammend, dargestellt und häufig mit Kriminalität und sexueller Gewalt in Verbindung gebracht.

Eine weitere Erschwernis hinsichtlich der strukturellen Integration im Vergleich zu anderen Asylwerber/innen-/Zuwander/innengruppen zeigt sich darin, dass afghanische Geflüchtete in Österreich die größte Kategorie an subsidiär Schutzberechtigten ausmachen. Beispielsweise gab es im Jahr 2017 insgesamt 3171 Gewährungen von subsidiärem Schutz an Afghan/inn/en. Die danach statistisch folgenden zweit- und drittgrößten Gruppen dieses Aufenthaltstitels sind Syrer/innen und Iraker/innen mit 1248 bzw. 1027 Gewährungen (vgl. BMI 2017). Dieser Status beinhaltet zwar das Erbringen von verpflichtenden Integrationsleistungen, der Aufenthalt in Österreich ist jedoch zeitlich beschränkt. Diese zeitliche Limitierung eines Aufenthalts in Österreich bringt Afghan/inn/en u.a. bei Arbeitgeber/inne/n und Vermieter/inne/n aufgrund der unsicheren Planbarkeit in eine nachteilige Ausgangslage, wie folgend auch in der empirischen Analyse dargelegt wird. Somit zeigt sich, dass sowohl die o.a. heterogenen Voraussetzungen, als auch der Zugang zu bestimmten Integrationsmaßnahmen ausschlaggebend für eine institutionelle Einbindung afghanischer Geflüchteter sind. In Anbetracht der aktuell medialen und gesellschaftlichen Diskriminierung von Afghan/inn/en erweist sich die Frage nach der Unterstützungsfunktion sozialer Beziehungen im Rahmen struktureller Integrationsprozesse als besonders relevant. Dabei haben sich anhand unseres empirischen Materials drei wesentliche Bereiche herauskristallisiert, die das Zusammenspiel von einer strukturellen Einbindung und den gegebenen sozialen Kontakten proklamieren und folgend einer näheren Analyse unterzogen werden: der Spracherwerb sowie die Integration am Arbeits- und Wohnungsmarkt.

\section{Erwerb der deutschen Sprache}

Der zügige Erwerb der deutschen Sprache wird sowohl aus staatlicher Perspektive als auch von Seiten der Gesprächspartner/innen dieses Forschungsprojektes als Ausgangsbasis für eine weitere Einbindung in die österreichische Gesellschaft und als wesentlich für das persönliche Wohlbefinden in Österreich betrachtet. Dabei sind insbesondere der Kontakt zu Personen aus der österreichischen Mehrheitsgesellschaft und die damit verbundene Möglichkeit, die bereits erworbenen Deutschkenntnisse im Alltag umzusetzen, relevant. Mit der Ankunft in Österreich und dem damit einhergehenden Status als Asylwerber/in sowie der Unterbringung in Asylunterkünften ist der 
Aufbau eines sozialen Netzwerks meist auf bestimme Personengruppen beschränkt. Die dort möglichen sozialen Kontakte setzen sich hauptsächlich aus Asylwerber/inne/n aus dem gleichen oder anderen Herkunftsländern sowie aus Sozialarbeiter/inne/n und teilweise Freiwilligen aus der Zivilgesellschaft zusammen. Vor allem letztere Gruppe stellt während der Zeit des Status als Asylwerber/innen, der sich teilweise über Jahre ziehen kann und während dessen kein Anspruch auf staatlich finanzierte Sprachkurse besteht, eine wichtige Ressource und Hilfestellung beim Spracherwerb dar.

I: Auf deinem erfolgreichen Weg haben dir eher die Österreicher/innen oder die Afghan/inn/en mehr geholfen?

B: Ich habe noch keine Hilfe von den Afghanen erhalten. Ganz am Anfang hat mir ein Afghane namens XY geholfen. Aber die Österreicher/innen haben mir sehr viel geholfen. Es waren Menschen dabei, die mir kostenlos die Sprache beigebracht haben, mit ihnen ich noch im Kontakt stehe und sie zuhause besuchen gehe oder wir gemeinsamen Sport machen. Im Flüchtlingsheim, wo ich früher war, haben sie uns öfter besucht und mit uns was unternommen und uns die Sehenswürdigkeiten der Stadt gezeigt und uns auch finanziell unterstützt. (I1, männlich, *1996, seit 2015 in Ö.)

Wie sich aus dem hier angeführten Beispiel zeigt, können durch das anfängliche freiwillige Engagement von Personen aus der österreichischen Mehrheitsgesellschaft auch freundschaftliche, längerfristige Beziehungen entstehen, die wiederum durch allägliche Kontakte und Freizeitaktivitäten den Spracherwerb und die soziale Einbindung in die österreichische Gesellschaft fördern. Deutsch auch in der Praxis anwenden zu können und etwa in weiteren von NGOs oder privaten Ehrenamtlichen organisierten Konversationsübungen zu vertiefen, wird als wesentliche Ergänzung zu den staatlich organisierten Sprachkursen eingeschätzt. Die Notwendigkeit dieser zusätzlichen Übungsmöglichkeit wurde von unseren Interviewpartner/inne/n sowohl mit dem positiven Lerneffekt der praktischen Anwendung als auch mit den langen Pausen zwischen den einzelnen Sprachkursmodulen begründet. Insbesondere betrifft dies Personen, die im ruralen Raum leben, da dort das Angebot an Sprachkursen wesentlich geringer ist als in den Städten. Auch für Frauen sind derartige Initiativen relevant, weil sie, aufgrund mangelnder Kinderbetreuungsstätten keine Möglichkeit haben an einem solchen Kursen teilzunehmen.

Wie bereits anhand der Unterbringung in Grundversorgungsquartieren erläutert wurde, haben die Wohnsituation und das soziale Umfeld des Wohnsitzes einen wesentlichen Einfluss auf den Erwerb der deutschen Sprache. Insbesondere die Respondent/ inn/en, die im ländlichen Raum leben, heben das Engagement der dortigen Bewohner/ innen sowie die dort unumgängliche Kommunikation mit der Bevölkerung als besonders sprachförderlich hervor. In größeren Städten wie Wien werden aufgrund der großstädtischen Anonymität und der geringeren Notwendigkeit der Kommunikation auf Deutsch eher Strategien wie die Inklusion in österreichische Familien oder in WGs mit Personen 
aus der österreichischen Mehrheitsgesellschaft als unterstützend für den Spracherwerb betrachtet.

Dennoch ist es wichtig zu betonen, dass für die meisten der Interviewpartner/ innen, neben Kontakten zu Personen aus der österreichischen Mehrheitsgesellschaft, Personen mit einem ähnlichen Erfahrungshintergrund hinsichtlich der Flucht und/oder aus der eigenen ethnischen Community eine wichtige Basis für das Wohlbefinden im neuen Lebenskontext darstellen. Der Faktor des persönlichen Wohlbefindens und des freundschaftlichen bzw. familiären Halts in schwierigen Situation darf bei Fragen der strukturellen Integration nicht außer Acht gelassen werden, da soziale Beziehungen generell ein wesentliches Auffangnetz darstellen, um überhaupt weitere Schritte der strukturellen Eingliederung konstruktiv beschreiten zu können.

\section{Arbeitsmarktintegration}

Neben dem Spracherwerb wird die möglichst rasche Integration in den Arbeitsmarkt sowohl aus staatlicher Perspektive als auch von den Respondent/inn/en des Forschungsprojektes selbst als der nahezu wichtigste Schritt der strukturellen Integration angesehen. Aus staatlicher Sicht bringt dies eine finanzielle Entlastung, für die Geflüchteten selbst die Möglichkeit auf eigenen Füßen zu stehen, einen gesellschaftlichen Beitrag zu leisten und sich eine Zukunftsperspektive aufzubauen.

B: [...] ,, wenn man eine Mutter ist, dann hat man den Stress, dass man sich denkt ... Gut, der Vater meiner Kinder kann nicht arbeiten ... “ Dass eines Tages zum Beispiel ... Dass, wenn uns der Staat Österreich eines Tages nicht helfen sollte, was ich dann tun sollte ... Diese Sache stresst mich immer. Wegen dieser Sache streite ich immer mit meinem Mann. Ich streite immer mit ihm und sag " ihm, dass er die Sprache lernen muss und er eine Arbeit finden muss. Ich möchte kein Sozialgeld mehr empfangen. Zum Beispiel ... Wie lange soll ich warten, dass heute der dritte des Monats ist und, dass da mein Geld kommt vom Sozialamt? Wie lange? Dann streiten wir uns und dann frage ich mich „,Wieso überhaupt? Wieso streitest du dich überhaupt mit ihm? Wieso bist du so dumm. Der Arme will doch eh arbeiten, aber kann einfach nicht. " (I2, weiblich, *1990, seit 2015 in Ö.)

Deutlich wird hier, dass mit dem Ziel auf eigenen Beinen stehen zu können und nicht auf staatliche Unterstützung angewiesen zu sein, ein starker sozialer Druck entsteht. Die finanzielle Unabhängigkeit einer Familie lässt sich meist nicht durch eine einzige arbeitende Person erreichen, sondern bedarf der Einbindung mehrerer Familienmitglieder. Damit einher geht die Erwartung des Spracherwerbs und die Ambivalenz zwischen schnellem Berufseinstiegs und zukunftsorientierter Ausbildung mit zunächst niedrigem oder keinem Gehalt, jedoch späteren Aufstiegschancen. Der Wunsch nach einer Integration in den Arbeitsmarkt ist damit kein individueller, sondern geknüpft an 
soziale Abhängigkeiten und Versorgungspflichten für Familienmitglieder im Aufnahmeals auch im Herkunftsland bzw. in den Transitländern.

Hinsichtlich der Arbeitsmarktintegration bestehen neben der bereits erwähnten Diskriminierung aufgrund des negativen öffentlichen Images und der Stereotypisierung „der Afghan/inn/en“, einige weitere Hürden, wie etwa mangelnde Deutschkenntnisse, nicht nachweisbare Qualifizierungen, Xenophobie oder auch gesundheitliche Probleme, welche den Einstieg in den Arbeitsmarkt erschweren. Institutionelle Beratungsangebote und Programme von staatlichen Organisationen, wie dem AMS oder den Wirtschaftskammern sowie von NGOs, sind dabei wichtige Anlaufstellen für offizielle Informationen zum österreichischen Arbeitsmarkt, Qualifizierungsmaßnahmen oder Nostrifizierungen von bereits erworbenen Abschlüssen. Wenn es jedoch zur Vermittlung von Arbeitsstellen kommt, berichten viele der Respondent/inn/en dieser Studie von negativen Erfahrungen mit staatlichen Institutionen. $\mathrm{Zu}$ nennen sind hier z.B. die Vermittlung von niedrig qualifizierten Beschäftigungen, um eine möglichst rasche Eingliederung zu erreichen, wenig Rücksichtnahme auf die realen Lebensumstände (z.B. Gesundheitszustand oder „Care“-Verantwortungen/Kinderbetreuung) sowie Demotivation anstatt ermutigender Anpassung der Erwartungen der Arbeitssuchenden an die realen Umsetzungsmöglichkeiten. Soziale Netzwerke erweisen sich daher in der Arbeitsmarktintegration sowohl hinsichtlich der Arbeitsvermittlung als auch als emotionale Unterstützung bei negativen Erfahrungen als besonders wichtig. Das folgende Beispiel veranschaulicht sowohl die Situation der Beratung durch das AMS als auch die essentielle Unterstützung durch das soziale Netzwerk.

I: Ja, dann, die Frage nach dem Arbeitsplatz. Wie haben Sie Ihren Arbeitsplatz gefunden? Das ist ja auch oft schwierig, eine Arbeit. Ist für Österreicher und für Ausländer natürlich oder Personen die ursprünglich vom Ausland sind, ja noch viel schwieriger oft.

B: Ja, das ist auch nicht so leicht. Es war so, ich hab' den Pflichtschule gehabt und danach musste ich arbeiten gehen. Und ich wollte Zahnarztassistentin werden oder Apothekerin. Und ich hab 'wirklich sehr lang gesucht und ich hab die Bewerbung auch mit meiner Freundin Christa geschrieben. Zu jeder Apotheke gegangen im 2. Bezirk und hab'ich nachgefragt und Bewerbung geschickt und auch zu mehreren Zahnärzte. Aber ich hab' die Lehre nicht gefunden und dann hab 'ich mich gedacht, werd' ich eine Ausbildung machen, damit ich arbeiten kann. Und es gab die Heimhilfeausbildung mit Job. Und da hab 'ich gedacht: ,Aha, wenn ich den Ausbildung mache, dann krieg ich auch vielleicht dort Job und kann ich auch ..."

I: Und das haben Sie über das AMS gefunden oder?

B: Über den AMS, ja ich hab' auch. Na ich hab' das eigentlich am Computer, das hab 'ich selber nachgeschaut, dass das gibt mit meiner Freundin Christa und dann, war ich im AMS und mein Betreuer hat dort gesagt: „Nein, wir zahlen das nicht. Das kostet so viel " usw. "Ich war selber dort allein und ein zweites 
Mal bin ich mit meiner Freundin Christa [lacht] dorthin gegangen und dann hat sie alles erzählt usw. und dann hat der Betreuer gesagt: ,Ja, ok, wir zahlen die Ausbildung. "Ich kann schon das machen. Und über den WAF, AMS ...

I: Ja, ja. Genau.

B: „Ja. Dann hab“ ich den Ausbildung gemacht und die haben bezahlt. “ (I3, weiblich, *1988, seit 2009 in Ö., Asylberechtigte)

Sichtbar werden hier das hohe eigene Engagement, eine Arbeitsstelle zu finden, sowie die Offenheit, die eigenen Erwartungen den Gegebenheiten anzupassen. Das anfängliche Scheitern der Zusammenarbeit mit dem AMS wurde letztendlich aufgrund der Unterstützung einer österreichischen Freundin in eine positive Richtung gelenkt. Ausschlaggebend ist deshalb primär die Hilfe durch das soziale Netzwerk. Dieses Netzwerk inkludiert unterschiedliche Personengruppen - meist Privatpersonen aus der österreichischen Mehrheitsgesellschaft, Mitarbeiter/innen von NGOs oder aus der eigenen ethnischen Community - wie die folgenden Interviewausschnitte verdeutlichen.

B: Als ich nach Österreich gekommen bin, habe ich erst ab 2006 oder 2007 eine Arbeit finden können, davor habe ich Sprachkurse besucht. Im Laufe der Zeit habe ich eine gute Freundin gefunden, die mich damals während der Sprachkurse betreut hat. Sie hat mich mal gefragt, ob ich arbeiten möchte. Ich sagte $i h r$, ,ja " und sie hat mich dann zu sich ins Büro geholt und hat mir eine Stelle empfohlen, mit welcher auch eine gewisse Art an Ausbildung miteinbezogen war, d.h. eine Arbeit mit der entsprechenden Ausbildung. Über diese Sozialarbeiterin habe ich dann eine Stelle in einem Hotel gefunden. Dort habe ich 6 Jahre durchgehend gearbeitet. (I4, weiblich, *1966, seit 2001 in Ö., Asylberechtigte)

I: Und dort, wo sie in der Küche als Abwäscherin gearbeitet haben, woher hatten Sie die Information, dass dort jemand gesucht wird?

B: Bei einer Geburtstagsparty ...

I: Von Österreichern?

B: „Ja, von Österreichern. Dort war ein Koch, der dort gearbeitet hat. An diesem Abend hat er dort gekocht. Und an diesem Abend hat mich mein Vermieter gefragt, ob ich dort aushelfen kann in der Küche und dass er mir auch ein wenig Geld geben wird. “ Ich habe gesagt: ,,Ja klar, mache ich gerne. “ „Im Untergeschoss von uns ist ein Raum, wo die Österreicher ihre Partys feiern, Weihnachten, Fasching oder Geburtstagspartys ... dieser Raum gehört zu einer Schule. Und er hat gesagt, dass es um 9 anfängt und bis um 12 am Abend dauert. Und dort habe ich diesen Koch kennengelernt. Er hat mich gefragt, ob ich arbeiten möchte und dass er viel Arbeit hat, die er mir anbieten kann. Und hat mir gesagt, dass ich mich jederzeit melden kann, wenn ich arbeiten möchte, weil sie immer jemanden suchen. Und dann habe ich mich 
sehr gefreut, dass ich eine Arbeit gefunden habe. Nachdem ich aber dreimal dort war, wurde ich sehr müde. Es war also sehr schwer. "(I5, weiblich, * 1990, seit 2015 in Ö., subsidiär Schutzberechtigte)

Neben dem wesentlichen Zusammenspiel der sozialen Integration, der damit verbunden Möglichkeit zum informellen Austausch, und der Arbeitsmarktintegration zeigt sich an den oben angeführten Beispielen auch die bereits angesprochene Problematik der Eingliederung in niedrigqualifizierte Arbeitsbereiche und Hilfsjobs. Wie jedoch aus dem Datenmaterial ebenfalls deutlich wurde, können diese Jobs eine erste Ausgangsbasis für weitere berufliche Entwicklungen und für finanzielle Selbstständigkeit sein, sofern das soziale Netzwerk und die institutionell angebotenen Beratungen und Weiterbildungsmaßnahmen ineinandergreifen.

Betrachtet man dieses Zusammenwirken überdies bei Personen, die sich noch im Asylverfahren befinden und bei denen die institutionalisierte Arbeitsvermittlung somit gänzlich wegfällt, so gewinnt die Bedeutung der sozialen Kontakte eine zusätzliche Tragweite gegenüber Personen mit Asylstatus, die Österreicher/inne/n hinsichtlich des Arbeitsmarktzugangs gleichgestellt sind. Eine Expertin erläutert dies in ihrer Aufgabe als Koordinatorin einer privaten Grundversorgungsunterkunft in Oberösterreich folgendermaßen:

I: Bezüglich Arbeitsmöglichkeiten, habt ihr da Kontakte? Mit dem AMS z.B.?

E: Das war eigentlich auch hauptsächlich über die Gemeinde. Der Bürgermeister war dann eh extra, den hab' ich schon erwähnt. Aber die Gemeinde war eigentlich auch ein sehr wichtiger Kooperationspartner. Die haben sich sehr bemüht. Die haben relativ schnell auch den Flüchtlingen Jobs angeboten und zwar so Straßenreinigungsarbeiten. Da kriegt man glaub ich 5 Euro in der Stunde. Das ist dieser offizielle Job, den man ohne Asylstatus machen darf. Das war cool, weil die alle motiviert waren. Also das waren nur die Männer. Und auch die vom anderen Haus. Da hat es dann so einen Plan gegeben, den hat die Gemeinde zusammen mit meinem Bruder erstellt. Also einen richtigen Wochenplan, wer welche Stunden wo eingeteilt ist. Da sind sie dann echt hingekommen zu den Straßenreinigungsarbeiten und sind dafür bezahlt worden. (E1, Koordinatorin einer privaten Grundversorgungsunterkunft)

Wie bereits anhand des Spracherwerbs dargestellt, wird auch hier deutlich, dass der Aufenthalt in einer ländlichen Gemeinde eine starke soziale Integration in die Ortsgemeinschaft mit sich bringt. Begründet wird dies unter anderem mit einer im Vergleich zu größeren Städten stärkeren sozialen Kohäsion und gegenseitiger Verpflichtungen der Bewohner/innen untereinander und damit auch gegenüber den Geflüchteten als neuen Bewohner/inne/n des Ortes. Mit der Bildung von Hilfsnetzwerken werden auch Geflüchtete nach der ersten Orientierungsphase oftmals zu aktiven Helfer/inne/n für Neuankommende und Bedürftige des Ortes. 


\section{Wohnintegration}

Wie bereits in der Analyse der beiden vorangegangenen Bereiche „Sprache“ und „Arbeit“ veranschaulicht wurde, besitzt der Wohnort einen wesentlichen Einfluss auf die Zugangsmöglichkeiten zu institutionellen Integrationsmaßnahmen wie Sprachkurse oder Beratungs-, Weiterbildungs- und Vermittlungsstellen wie dem AMS, aber insbesondere auch auf die soziale Integration.

E: Auch ganz viele Leute aus Afghanistan erzählen uns, dass sie das schon mitbekommen, dass dieses negative Bild, das es halt von Leuten aus Afghanistan gibt, dass es das für sie halt auch schwieriger macht, nur weil sie aus Afghanistan kommen, dann ein WG-Zimmer zu finden. (E2, Mitarbeiterin einer NGO, WG-Vermittlungsplattform)

Es zeigt sich jedoch besonders bei Personen afghanischer Herkunft, u.a. aufgrund des negativen öffentlichen Bildes und der kriminalisierten Darstellung dieser Personengruppe sowie aufgrund des beschränkten Aufenthaltes durch den Status als subsidiär Schutzberechtigte folgendes: Der Wohnort ist nicht nur wesentlich für die soziale Integration, sondern das soziale Netzwerk ist ebenso ausschlaggebend dafür, eine geeignete Unterkunft auf dem freien Wohnungsmarkt zu finden.

Die Wohnintegration - also die Inklusion in reguläre Wohnverhältnisse zur Vermeidung von Segregation und Exklusion - und die Möglichkeit der freien Wohnortentscheidung beginnen mit dem Erhalt eines positiven Asylbescheids (als Asyl- oder subsidiär Schutzberechtigte/r) und dem damit einhergehenden Verweis aus dem Grundversorgungsquartier für Aslywerber/innen. Für den Bereich „Wohnen“ greifen keine staatlichen Integrationsmaßnahmen und die Wohnungssuchenden sind mit häufig noch unzureichenden Sprachkenntnissen ${ }^{4}$ auf sich allein gestellt. Bei der Überbrückung der Sprachbarrieren helfen oft Personen aus der eigenen ethnischen Community, welche schon länger in Österreich sind, z.B. bei der Kontaktierung von Vermieter/ inne/n, bei Antragstellung und Übersetzungstätigkeiten. Zu den Sprachbarrieren kom men häufig finanzielle Schwierigkeiten, da kaum Ersparnisse angehäuft werden konnten und damit folglich der Ausschluss von günstigeren Wohnmöglichkeiten wie Genossenschaftswohnungen gegeben ist, aber insbesondere bestehen Probleme bezüglich des Aufbringens von hohen Kautionen.

B: Genossenschaftswohnungen sind ja sehr teuer, dort muss man um die 10.000 $-15.000 €$ im Vorhinein schon zahlen, ein Geld, das wir noch nicht haben. Und bei der Gemeinde ist es so, dass man dort zwei Jahre lang angemeldet sein muss. Das waren wir zwar, aber dann hat man uns an die Wohnkommission weitergeleitet. Dort hat man uns gesagt, dass sie keine Wohnung für uns haben und jetzt müssen wir einfach warten.

\footnotetext{
${ }_{4}$ Institutionalisierte Sprachkurse sind auch erst nach Erhalt des positiven Asylbescheids zugänglich.
} 
I: Gut, dass sie die Wohnung ansprechen, als sie nach Österreich kamen, wo haben Sie da gelebt? Haben Sie in einem Asylheim gelebt?

B: Mein Mann ist ja vor uns hierhergekommen, und er hat diese Einzimmerwohnung gefunden. Er hatte gedacht, dass es schneller gehen würde, und wir bald eine größere Wohnung finden würden, aber es hat sich nichts ergeben. Und seitdem ich da bin, lebe ich mit meiner Familie in dieser Einzimmerwohnung. [...]

Das Problem ist, dass man außer bei Gemeindewohnungen, sonst immer eine große Anzahlung leisten muss. Mein Mann arbeitet zwar und verdient normales Geld, womit wir unser Leben leisten können, aber es ist nicht so viel, dass wir solche Summe zahlen könnten. Wir haben nichts ansparen können. Vor sieben Monaten haben wir den positiven Asylbescheid erhalten, davor waren wir Asylwerber. Damals hatten wir nur das Gehalt von meinem Mann, und wir haben kein Kindergeld erhalten. Mit seinem Geld mussten wir die Miete und andere Kosten bezahlen. Somit konnten wir nichts auf die Seite legen. Bei der Gemeinde ist es so das man 5 bis 6 Jahre warten muss, und in 5 bis 6 Jahren sind meine Kinder schon fast erwachsen. Sie können so lange nicht mit uns in einem Zimmer leben. Die Gemeinde sagt auch, wir sollen sechs Jahre warten. Die Wohnungen, die man als Option erhält, sind manchmal sehr alt oder sehr kalt. (I7, weiblich, ${ }^{*} 1990$, seit 2015 in Ö., Asylberechtigte)

Neben den erwähnten Schwierigkeiten, die finanziellen Mittel für eine Genossenschaftswohnung aufzubringen, wird daraus auch die Problematik des Zugangs zu günstigen Gemeindewohnungen ersichtlich. Für den Antrag auf eine Gemeindewohnung ist der Nachweis des Hauptwohnsitzes in der jeweiligen Gemeinde notwendig. Die Dauer der Nachweispflicht variiert, in Wien muss beispielsweise ein mindestens zweijähriger Hauptwohnsitz nachgewiesen werden, um überhaupt einen Antrag stellen zu können. Nachdem der Antrag gestellt wurde, können bis zur Zuteilung einer geeigneten Gemeindewohnung Jahre vergehen. Da die meisten Asylberechtigten nach dem positiven Bescheid die Asylunterkunft verlassen und so schnell wie möglich eine leistbare Unterkunft finden müssen, führt dies häufig zu prekären Wohnsituationen. Die Interviewpartnerin, von der das obige Zitat stammt, lebt derzeit mit ihren zwei Kindern und ihrem Mann in einer Einzimmerwohnung, andere Optionen, die mit den vorhande-nen finanziellen Mitteln möglich wären, entsprechen häufig nicht den Bedürfnissen einer jungen Familie. Oftmals wird die Notlage der Wohnungssuchenden, in kürzester Zeit ein Dach über dem Kopf zu finden, von Vermieter/inne/n ausgenutzt und für kleinsten Wohnraum und geteilte Zimmer werden horrende Mieten verlangt.

[...] Ich glaube das Schlimmste, was uns bzw. den Menschen, die in derselben Lage sind wie wir passieren kann, ist es ... Es gibt Menschen, die eine Art Verbindung zwischen den Vermietern und den Personen, die eine Wohnung suchen, stellen. (Makler sind gemeint) Diese finden ... gut manche Wohnungen sind ganz in Ordnung ... Für manche Wohnungen benötigt man eine Arbeits- 
bestätigung. Man benötigt eine Bestätigung, dass man irgendwo beschäftigt ist. Und diese sagen dann, dass es kein Problem ist, wenn man so eine Bestätigung nicht hat. Diese verlangen, aber dann von der Person, die auf Wohnungssuche ist, fast 2.000 bis 5.000 Euro, um eine Wohnung zu bekommen. Unserer Meinung nach ist das sehr unverschämt. Deshalb haben wir das nicht gemacht. Wir haben uns direkt mit einem Österreicher in Verbindung gesetzt, der dem Vermieter bestätigt hat, dass wir in der Lage sind, die Miete zu zahlen. Und aus diesem Grund möchte unser Vermieter alle drei Monate von uns die Miete bekommen, wegen seiner eigenen Sicherheit. Es ist aber sehr schwer eine Arbeit zu finden, weil man eine Arbeitsbestätigung braucht und jemand aus der Familie arbeiten muss. Aus diesem Grund ... Ich habe von vielen anderen gehört, dass eine Person zum Beispiel eine Gemeindewohnung gefunden haben und dann betrogen wurden. Es sind viele Probleme entstanden. Und ich finde, dass die Menschen hier es verstehen sollten, dass man nicht sofort arbeiten kann. Oder man sofort einen Job finden kann, aber dass man trotzdem irgendwo leben muss. Das ist meiner Meinung nach ein großes Problem. Und die Wohnungssuche war auch für uns sehr schwer. (I8, weiblich, *1999, seit 2015 in Ö.)

Die Unterstützung durch Personen aus dem sozialen Netzwerk - dies inkludiert Privatpersonen unterschiedlicher Gesellschaftsgruppen, also auch Mitarbeiter/innen von NGOs - beim Finden einer geeigneten Wohnung ist daher maßgeblich. Hilfestellungen reichen von der Zurverfügungstellung von Privatwohnungen durch Personen der österreichischen Mehrheitsgesellschaft über die Unterstützung bei der Suche bis zur finanziellen Hilfe durch Zuschüsse oder Bürgschaften meist von Personen aus der eigenen ethnischen Community.

I: Und die Menschen, die Ihnen geholfen haben, waren nur Ihre Freunde?

B: Ja, genau.

I: Haben Sie vorhin die Caritas auch erwähnt? Oder nicht?

B: Caritas hat nur ... Ich habe zum Beispiel Geld von der Caritas erhalten, aber wegen der Wohnung, da haben wir nur von unserem Freund, den wir im Camp kennengelernt haben und der vor uns den Pass erhalten hat und nach Wien kam, Hilfe erhalten. Sonst haben wir von keiner Organisation Hilfe erhalten. (I9, weiblich, *1985, seit 2011 in Ö.)

Projekte von NGOs können zudem dazu beitragen einerseits direkt vermittelnd tätig zu sein und andererseits Kontakte zwischen engagierten Personen der österreichischen Mehrheitsgesellschaft und geflüchteten Wohnungssuchenden herzustellen, welche wiederum bei der Wohnungssuche unterstützen können.

E: Auch diese Workshops, da entstehen ja auch so eine Art Buddy-Beziehungen. Es ist immer so, dass ein Geflüchteter mit einem Ehrenamtlichen gemeinsam 
eine WG sucht und diesen Bewerbungstext zusammen verfasst und auf den Plattformen schauen, wo man was machen kann. Und das Ziel ist auch, dass die dann weiter in Kontakt bleiben und gemeinsam zu den WG-Castings gehen. Es ist jetzt nicht so, dass die ehrenamtliche Person dafür zuständig ist, dass die Person ein WG-Zimmer findet, aber es sollten ein bisschen langfristigere Bindungen sein. (E3, Mitarbeiter einer NGO, WG-Vermittlungsplattform)

Insgesamt zeigt sich vor allem im Bereich „Wohnen“ das ineinandergreifende Zusammenwirken sozialer Netzwerke und struktureller Integration. Denn Soziale Kontakte sind ausschlaggebend für das Finden einer geeigneten Wohnsituation, die im Weiteren auch wesentlich zur sozialen Integration im neuen Lebenskontext beiträgt.

\section{Conclusio}

Der Bedarf an institutionsübergreifenden Maßnahmen zur Integration von geflüchteten Personen (mehrheitlich aus Syrien, Irak und Afghanistan) in Österreich wurde spätestens mit der Migrationsbewegung im Jahr 2015 verdeutlicht. Gesetzlich festgeschriebene Integrationsstrategien, die häufig politisch geleiteten Reformen obliegen, sollen dazu beitragen, die Einbindung Geflüchteter in ein gesellschaftliches Zusammenleben in Österreich zu fördern. Der Zugang zu sogenannten Integrationsmaßnahmen hinsichtlich Arbeitsmarktintegration, Spracherwerb und Alphabetisierung sowie der Vermittlung von Werte- und Orientierungswissen ist auf Geflüchtete mit Aufenthaltsstatus beschränkt. Demnach sind Asylwerbende in ihren Rechten im Ver-gleich zu Asylberechtigten und subsidiär Schutzberechtigten stark eingeschränkt. Jedoch veranschaulicht unsere qualitative Studie auf Basis des Samples afghanischer Geflüchteter, dass nicht nur Personen ohne Status, sondern auch jene mit legaler Aufenthaltsberechtigung mit großen Schwierigkeiten bei institutionellen Eingliederungsprozessen konfrontiert sind. Heterogene Voraussetzungen charakterisieren individuelle Integrationsverläufe und klassifizieren geflüchtete Afghan/inn/en als „Problemgruppe“ im Rahmen einer strukturellen Assimilation (vgl. Gordon 1964). Unsere Analyse verdeutlicht, dass in den drei wesentlichen Bereichen, Arbeitsmarktund Wohnintegration sowie Spracherwerb, die staatlich reglementierten Maßnahmen nicht ausreichend zu einer institutionellen Einbindung beitragen. Vielmehr wird veranschaulicht, dass soziale Kontakte bzw. Beziehungen zu Personen aus der österreichischen Mehrheitsgesellschaft sowie zu Personen aus der ethnischen Community und das daraus resultierende soziale Kapital als ausschlaggebender Faktor für den Beginn eines strukturellen Integrationsprozesses verzeichnet werden können. Demnach kann der von der österreichischen Gesetzgebung definierte Integrationsbegriff, der mit einer strukturellen Assimilation von Migrant/inn/en eingeleitet wird und wie im Integrationsschema von Esser (2001) dargestellt, nicht als linearer Prozess verstanden werden. 
Unsere Analyse zeigt vielmehr das Zusammenwirken und reziproke Verhältnis von struktureller und sozialer Integration. Soziale Beziehungen zu Personen aus der österreichischen Mehrheitsgesellschaft sowie zu Personen aus der ethnischen Community zu fördern und bei der Analyse von Integrationsmaßnahmen miteinzubeziehen manifestiert sich daher als besonders relevant.

\section{Literatur}

Ager A. \& Eyber C. (2002), Indicators of Integration. A Review of Indicators of Refugee Integration. Report to Home Office on behalf of Michael Bell Associates.

Ager A. \& Strang A. (2008), Understanding Integration. A Conceptual Framework. Journal of Refugee Studies, 21 (2), Oxford Press, pp. 166-191.

AMS - ArbeitsmarkTSERvice (ed.) (2017), Beschäftigung von AsylwerberInnen. Wien.

Anhut R. \& Heitmeyer W. (2000), Desintegration, Konflikt und Ethnisierung. Eine Problemanalyse und theoretische Rahmenkonzeption. In: Anhut R. \& Heitmeyer W. (eds.), Bedrohte Stadtgesellschaft. Konflikt- und Gewaltforschung. Weinheim/München, Juventa, pp. 1775.

Aschauer W. \& Gann F. (2017), Zu den Paradoxien der gegenwärtigen Integrationsdiskurse von Flüchtlingen in Österreich: Wie in der Forderung nach individueller Leistung erschwerte Ausgangs-und Kontextbedingungen missachtet werden. In: OBerlechner et. al., (eds.), Migration bildet. Baden-Baden, Nomos, pp. 279-302.

AsYLKOORDINATION ÖSTERREICH (ed.) (2017), Flüchtlinge \& Arbeit: Infoblatt der Asylkoordination Österreich (7), Wien.

BMEIA - Bundesministerium für Europa, Integration und Äusseres (ed.) (2015), 50 PunktePlan zur Integration von Asylberechtigten und subsidiär Schutzberechtigten in Österreich. Wien.

BMi - Bundesministerium für InNeres (ed.) (2017), Vorläufige Asylstatistik Dezember 2017. Wien.

Boyd M. \& NowaK J. (2012), Social Networks and International Migration. In: Martiniello, M. \& Rath J. (eds.), An Introduction to International Migration Studies. European perspectives. Amsterdam, University Press, pp. 79-106.

Castles et al. (2002), Integration: Mapping the Field. Report. Centre for Migration and Policy Research and Refugee Studies Centre, University of Oxford.

Deindl C. (2005), Soziale Netzwerke und soziales Kapital. Einfluss auf Lebenszufriedenheit und Vertrauen. Diskussionspapier der Forschungsgruppe Arbeit, Generation, Sozialstruktur (AGES) der Universität Zürich.

Dewalt K. \& Dewalt B. (2011), Participant observation: a guide for fieldworkers. Lanham, Alta Mira Press.

Elwert F. (2015), Religion als Ressource und Restriktion im Integrationsprozess. Eine Fallstudie zu Biographien freikirchlicher Russlanddeutscher. Wiesbaden, Springer.

Esser H. (2001), Integration und ethnische Schichtung. Arbeitspapier, 40, Mannheimer Zentrum für Europäische Sozialforschung. 
EXPERTENRAT FÜR INTEGRATION (ed.) (2016), Integrationsbericht. Integration von Asylberechtigten und subsidiär Schutzberechtigten in Österreich - Wo stehen wir heute? Zwischenbilanz des Expertenrats zum 50 Punkte-Plan, Wien.

EXPERTENRAT FÜR INTEGRATION (ed.) (2017), Integrationsbericht 2017. Flüchtlingsintegration bilanzieren - Regelintegration wieder thematisieren. Wien, Bundesministerium für Europa, Integration, Äußeres.

FARwick A. (2009), Migrantenquartiere - Ressource oder Benachteiligung? In: Gans P. (ed.), Räumliche Auswirkungen der internationalen Migration, Forschungsbericht der ARL 3. Hannover 2014, pp. 219-238.

FREY E. (2016), „Die Leser nicht deppert sterben lassen“, KOMMENTAR DER STANDARDREDAKTION, derstandard.at, 22.01.2016. $<$ https://derstandard.at/2000029593722/> [aufgerufen am 10.11.2018]

Gordon M. (1964), The Nature of Assimilation. In: Gordon M. (ed.), Assimilation in American Life The role of race, religion and national origins. New York, Oxford University Press, pp. 60-83.

Granovetter M.S. (1973), The Strength of the Weak Ties. American Journal of Sociology, 78 (6), pp. 1360-1380.

Han P. (2016), Soziologie der Migration. Konstanz/München, UVK Verlagsgesellschaft.

HANS S. (2016), Theorien der Integration von Migranten - Stand und Entwicklung. In: BRINKMANN H.U. \& SAUER M. (eds.), Einwanderungsgesellschaft Deutschland. Wiesbaden, Springer, pp. 24-50.

Haug S. (2007), Soziales Kapital als Ressource im Kontext von Migration und Integration. In: LÜDICKE J. (ed.). Soziale Netzwerke und soziale Ungleichheit: zur Rolle von Sozialkapital in modernen Gesellschaften. Wiesbaden, Springer VS, pp. 85-112.

KoHLBACHER J. (2017), Steps on the way to social integration: initial social interactions of refugees from Syria, Iraq and Afghanistan with the host society, their relevance, assessment and implications. In: Kohlbacher J. \& Schiocchet L. (eds.). From Destination to Integration Afghan, Syrian and Iraqi Refugees in Vienna. Wien, Verlag der Österreichischen Akademie der Wissenschaften, pp. 167-193.

KRUSE J. (2015), Qualitative Interviewforschung. Weinheim/Basel, Beltz Juventa.

LANDESKORRRESPONDENZ MEDIENINFO (ed.) (2017), Aktuelle Zwischenbilanz der Integration am Arbeitsmarkt in OÖ - Immer mehr Asylwerbende in OÖ können in jenen Jobs, die frei zugänglich sind, Fuß fassen. Zahlen, Praxisbeispiel: Dienstleistungsscheck, Forderungen. Amt der OÖ Landesregierung, Linz.

Lewis-Beck M.S., Bryman A. \& Liao T. (eds.) (2004), The SAGE Encyclopedia of Social Science Research Methods (Volume 1). Thousand Oaks/London/New Delhi, Sage.

Lin N. (2001), Social Capital: A Theory of Social Structure and Action. Cambridge.

Lockwood D. (1964), Social Integration and System Integration. In: Zollschang, G. \& Hirsch, W.(eds.), Explorations in Social Change. London, Routledge \& K. Paul, pp. 244-257.

Musterd S. (2003), Segregation and Integration. A contested relationship. Journal of Ethnic and Migration Studies, 29 (4), pp. 623-64.

ÖIF - ÖSTERREICHISCHER INTERGRATIONSFONDS (ed.) (2017), Informationen zum Integrationsgesetz, Wien.

RAusCHER H. (2016), „Probleme mit Afghanen sinnvoll lösen“, derstandard.at, 9.12.2016. $<$ https://derstandard.at/2000049072276/Probleme-mit-Afghanen-sinnvoll-loesen> [aufgerufen am 10.11.2018] 
Reinhold G. (ed.) (2000), Soziologie - Lexikon. München, Oldenbourg Verlag, 4. Auflage.

Rosenberger S. (2011), Integration von AsylwerberInnen? Zur Paradoxie individueller Integrationsleistungen und staatlicher Desintegration. In: Dahlvik J., Fassmann H. \& Sievers W. (eds.), Migration und Integration - wissenschaftliche Perspektiven aus Österreich. Jahrbuch 1/2011, Universität Wien, pp. 91-106.

STATistik Austria (ed.) (2018), Bevölkerung am 1.1.2018 nach detaillierter Staatsangehörigkeit und Bundesland.

$<$ https://www.statistik.at/web_de/statistiken/menschen_und_gesellschaft/bevoelkerung/ bevoelkerungsstruktur/bevoelkerung_nach_staatsangehoerigkeit_geburtsland/index.html> [aufgerufen am 13.11.2018]

Szigetvari A. (2018), „Regierung fixiert Ende der Lehre für Asylwerber“, derstandard.at, 26.08.2018.

$<$ https://derstandard.at/2000086070416/FPOe-will-Zugang-zur-Lehre-fuer-Asylwerbernwieder-verbieten $>$ [aufgerufen am 09.09.2018]

Wets J. (2006), The Turkish Community in Austria and Belgium. The Challenge of Integration. Turkish Studies, 7 (1), pp. 85-100.

Williams L. (2006), Social Networks of Refugees in the United Kingdom: Tradition, Tactics and New Community Spaces. Journal of Ethnic and Migration Studies, 32 (5), pp. 865-879.

\section{Respondent/inn/enliste}

I1, männlich, *1996, seit 2015 in Ö.

I2, weiblich, *1990, seit 2015 in Ö., subsidiär Schutzberechtigte

I3, weiblich, *1988, seit 2009 in Ö., Asylberechtigte

I4, weiblich, *1966, seit 2001 in Ö., Asylberechtigte

I5, weiblich, *1990, seit 2015 in Ö., subsidiär Schutzberechtigte

I6, weiblich, *1992, seit 2008 in Ö., Asylberechtigte

I7, weiblich, *1990, seit 2015 in Ö., Asylberechtigte

I8, weiblich, *1999, seit 2015 in Ö., subsidiär Schutzberechtigte

I9, weiblich, *1985, seit 2011 in Ö., subsidiär Schutzberechtigte

E1, Koordinatorin einer privaten Grundversorgungsunterkunft

E2, Mitarbeiterin einer NGO,WG-Vermittlungsplattform

E3, Mitarbeiter einer NGO,WG-Vermittlungsplattform 